\title{
Medicamentos fitoterápicos utilizados para constipação intestinal comercializados em uma drogaria da cidade de Manaus - AM
}

\author{
Herbal Medicines used for constipation commercialized in a drugstore in the city of Manaus - AM \\ Hierbas Medicinales utilizadas para el estreñimiento comercializadas em uma farmácia de la \\ ciudad de Manaus - AM
}

Recebido: 09/11/2021 | Revisado: 18/11/2021 | Aceito: 24/11/2021 | Publicado: 26/11/2021

\author{
Júlia Costa Silva \\ ORCID: https://orcid.org/0000-0002-1122-7169 \\ Centro Universitário do Norte, Brasil \\ E-mail: silvajulia.2604@gmail.com \\ Tamyle Barros Figueiredo \\ ORCID: https://orcid.org/0000-0003-2353-8009 \\ Centro Universitário do Norte, Brasil \\ E-mail: tamylebarros@gmail.com \\ Vívian Moreira da Silva \\ ORCID: https://orcid.org/0000-0002-7909-4833 \\ Centro Universitário do Norte, Brasil \\ E-mail: vivianms19@gmail.com \\ Marcos Túlio da Silva \\ ORCID: https://orcid.org/0000-0002-7653-4944 \\ Faculdade Uninorte, Brasil \\ E-mail: marcostulio39@gmail.com
}

\begin{abstract}
Resumo
Objetivos: Este estudo tem como principal objetivo identificar através de levantamento quantitativo e descritivo quais são os medicamentos fitoterápicos mais vendidos e utilizados para o tratamento de constipação comercializados por uma drogaria na cidade de Manaus. Métodos: listar quais os medicamentos fitoterápicos para constipação foram vendidos em agosto de 2019 a agosto de 2020; apresentar os tipos de medicamentos fitoterápicos que agem na constipação intestinal; listar os medicamentos que foram vendidos para a constipação intestinal e pontuar os resultados se utilizados de forma racional. Este trabalho trata-se de uma pesquisa exploratória e descritiva realizada em uma drogaria localizada na cidade de Manaus, Amazonas. Resultados: os medicamentos fitoterápicos mais vendidos para a constipação intestinal foi o medicamento com as plantas medicinais Senna alexandrina e Cassia fistula com 40\% (Laboratório A), logo em seguida o que continha a planta medicinal Plantago ovata (35\%), Senna alexandrina e Cassia fistula (Laboratório B) (13\%) e Aloe ferox e Gentiana lutea (12\%), entre agosto de 2019 a agosto de 2020. Conclusão: o uso irracional dos fitoterápicos causam efeitos colaterais adversos, no entanto com a dosagem adequada os resultados são positivos.
\end{abstract}

Palavras-chave: Fitoterápicos; Constipação; Comercialização.

\begin{abstract}
Objectives: This study has as main objective to identify through quantitative and descriptive survey which are the most sold herbal medicines used for the treatment of constipation marketed by a drugstore in the city of Manaus. Methods: list which herbal medicines for constipation were sold in August 2019 to August 2020; present the types of herbal medicines that act on constipation; list the medicines that were sold for constipation and score the results if used rationally. This work is an exploratory and descriptive research conducted in a drugstore located in the city of Manaus, Amazonas. Results: the most sold herbal medicine for intestinal constipation was the medicine with the medicinal plants Senna alexandrina and Cassia fistula with $40 \%$ (Laboratory A), right after that which contained the medicinal plant Plantago ovata (35\%), Senna alexandrina and Cassia fistula (Laboratory B) (13\%) and Aloe ferox and Gentiana lutea (12\%), between August 2019 to August 2020. Conclusion: irrational use of herbal medicines cause adverse side effects, however with proper dosage the results are positive.
\end{abstract}

Keywords: Herbal medicines; Constipation; Marketing.

\section{Resumen}

Objetivos: Este estudio tiene como objetivo principal identificar a través de una encuesta cuantitativa y descriptiva cuáles son las hierbas medicinales más vendidas para el tratamiento del estreñimiento comercializadas por una droguería en la ciudad de Manaus. Métodos: enumerar qué medicamentos a base de hierbas para el estreñimiento se vendieron en 
agosto de 2019 a agosto de 2020; presentar los tipos de medicamentos a base de hierbas que actúan sobre el estreñimiento; enumerar los medicamentos que se vendieron para el estreñimiento y puntuar los resultados si se utilizan racionalmente. Este trabajo es una investigación exploratoria y descriptiva realizada en una farmacia ubicada en la ciudad de Manaus, Amazonas. Resultados: el fitoterápico más vendido para el estreñimiento intestinal fue el medicamento con las plantas medicinales Senna alexandrina y Cassia fistula con $40 \%$ (Laboratorio A), inmediatamente después del que contenía la planta medicinal Plantago ovata (35\%), Senna alexandrina y Cassia fistula (Laboratorio B) (13\%) y Aloe ferox y Gentiana lutea (12\%), entre agosto de 2019 a agosto de 2020. Conclusión: el uso irracional de los medicamentos a base de hierbas causa efectos secundarios adversos, sin embargo, con una dosis adecuada los resultados son positivos.

Palabras clave: Medicamentos a base de hierbas; Estreñimiento; Comercialización.

\section{Introdução}

O Brasil possui uma diversidade em espécies de plantas, concentrada em grande parte na Floresta Amazônica, que são muito utilizadas na fabricação de produtos medicinais ou medicamentos fitoterápicos (Brasil, 2016).

Dentre os tipos de plantas medicinais utilizadas para a fabricação de medicamentos fitoterápicos pode-se citar o uso de Senna alexandrina, que pode ser popularmente conhecida como Sene, sua estrutura é de arbusto com crescimento fácil e rápido, sendo suas folhas e sementes utilizadas pela indústria farmacêutica para a fabricação de medicamentos voltados para o tratamento da constipação (Severo et al., 2018; Vilanova-Sanchez et al.,2018).

Com relação à constipação, esta é uma das principais queixas que comprometem a qualidade de vida de diversas pessoas, sendo responsável por cerca de 2,5 milhões de visitas ao médico por ano, e motivo de altos custos com médico e medicamentos no mundo todo. Nas pesquisas apresentadas, o público feminino é o que mais sofre com essas queixas de constipação intestinal por causa de questões fisiológicas voltadas para a produção de estrogênio e progesterona que atua diretamente no funcionamento gastrointestinal voltado para a ativação do sistema nervoso autônomo (Dantas, 2019; Diaz, Bittar, Mendez, 2021; Jin et al., 2021).

Com isso, a constipação está dividida em primária e secundária. A primária está voltada para a regulação desordenada voltada para os componentes neuromusculares entre o cólon, reto e ânus. A secundária está relacionada com vários fatores que distúrbios neurológicos, doenças de Parkinson, distúrbios endócrinos e metabólicos (Cieza et al.,2017; Basilisco \& Coleta, 2016).

Assim, a constipação pode também ser considerada como uma condição dispendiosa que pode afetar a vida da pessoa em todas as esferas, como no desempenho das atividades laborais como nas atividades diárias. A dificuldade de evacuação, sensação de mal-estar ou de movimentos frequentes abdominais, causa desconforto em diversas pessoas e está presente na vida da maioria delas a cada 3 ou 4 dias, por semana (Thompson et al.,2020; Huang et al., 2017).

Com isso, esses sintomas acabam comprometendo atividades sociais ou físicas, de maneira que a sensação desconfortante compromete o bem-estar físico de todos as pessoas que sentem esses sintomas, adentrando na sua produtividade laboral (Bharucha et al.,2016; Longstreth et al., 2016)

Dessa forma, o estudo voltado para os medicamentos fitoterápicos para o tratamento de constipação é de extrema relevância pela população pois envolve saúde pública, devido a quantidade de casos de constipação e a busca por um resultado imediato ou a curto prazo. Entende-se que alguns dos pacientes que fazem uso de medicamentos fitoterápicos para a constipação também fazem uso de outros medicamentos, por isso se torna importante o conhecimento sobre a ação desses medicamentos mesmo que sejam considerados naturais, pois se forem usados de forma irracional e sem orientação adequada também apresentam reações adversas no corpo do paciente e se este possui um histórico de outros medicamentos ou comorbidades pode agravar, caso seja realizado de forma incorreta o seu uso (Rad, 2017;Colette et al.,2017; Cirillo \& Capasso, 2015).

Diante isto, este estudo tem como principal objetivo de identificar através de levantamento quantitativo e descritivo quais são os medicamentos fitoterápicos mais vendidos e utilizados para o tratamento de constipação comercializados por uma 
drogaria na cidade de Manaus. Para isso, busca-se apresentar os tipos de medicamentos fitoterápicos que agem na constipação intestinal como regulador; descrever como ocorre o mecanismo de ação dessas plantas medicinais utilizadas para o tratamento de constipação intestinal presentes nos medicamentos fitoterápicos; pontuar os casos eficientes para constipação intestinal com o uso de fitoterápicos de plantas medicinais apresentados por outros pesquisadores internacionais.

\section{Metodologia}

Trata-se de um estudo exploratório onde foi realizada pesquisa em drogaria localizada na cidade de Manaus, aplicando-se técnicas voltadas para análises documentais e observacionais no sistema de vendas comerciais do respectivo local. Assim, pode-se afirmar que a pesquisa exploratória reúne informações importantes sobre um determinado local ou objeto estudado que serve para delimitar toda a pesquisa (Severino, 2018). Com base nisso, a pesquisa exploratória tem como finalidade reunir dados e informações sobre o objeto de estudo com o intuito de buscar conhecer algum fenômeno ou obter deste total conhecimento (Pereira, 2018).

Com isso, foi realizado uma pesquisa com abordagem qualiquantitativa. Este tipo de pesquisa é caracterizado por "ser mensurados em números, classificados e analisados utilizando-se de técnicas estatísticas" (Dalfovo, et al., 2008;). Portanto, realizou-se uma pesquisa descritiva, observacional e documental, realizando-se análises no programa de controle de estoque em uma drogaria na cidade de Manaus.

Contudo, para a seleção da relação de fitoterápicos foram usados critérios de inclusão e exclusão. Para inclusão, foram incluídos na pesquisa todos os fitoterápicos para constipação vendidos como reguladores entre agosto de 2019 a agosto de 2020. Desse modo, foram descartados, todos outros medicamentos ou fitoterápicos que não estão de acordo com os objetivos da pesquisa, ou que foram vendidos antes de 2019.

\section{Resultados e Discussão}

De acordo com os critérios discriminados para a seleção dos dados a serem apresentados nesta pesquisa, primeiramente encontrou-se na relação de dados 956 vendas de medicamentos com prescrição, logo em seguida encontrou-se a relação de fitoterápicos e depois os medicamentos que tiveram saída no sistema, mas que não se encontrou nos registros as prescrições por motivos diversos, sejam por não precisar de medicamentos ou por extravio da receita.

Figura 1 - Coleta de dados do levantamento da pesquisa na drogaria.
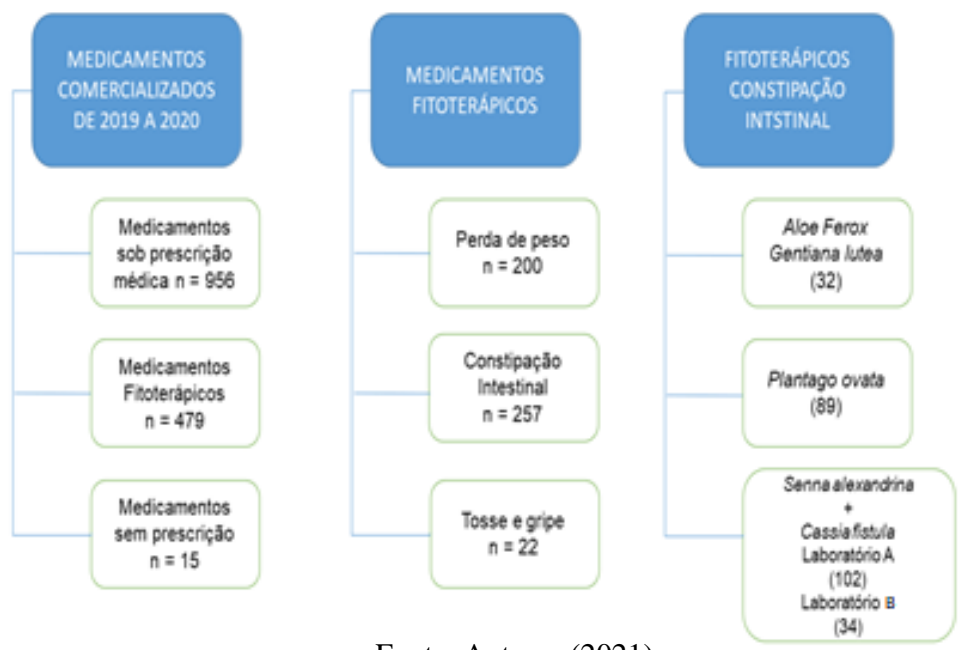

Fonte: Autores (2021). 
Tabela 1 - Relação dos fitoterápicos vendidos em ago/2019 à ago/2020.

\begin{tabular}{|c|c|c|}
\hline Espécie de planta & Forma Farmacêutica & Qte \\
\hline $\begin{array}{l}\text { Gentiana lutea (rizoma e raiz); } \\
\text { Aloe } \quad \text { ferox (suco dessecado } \\
\text { das folhas). }\end{array}$ & $\begin{array}{c}\text { Extrato mole de Gentiana } \\
\text { lutea }(4,0 \mathrm{mg} / \mathrm{mL}) ; \\
\text { Extrato fluido de Aloe } \\
\text { ferox } \\
(0,18 \mathrm{~mL} / \mathrm{mL}) .\end{array}$ & 32 \\
\hline $\begin{array}{c}\text { Casca da semente de Plantago } \\
\text { ovata Forssk } \\
\text { (Psyllium } \\
\text { husk })\end{array}$ & $\begin{array}{l}\text { Pó para preparações } \\
\text { extemporâneas, sem } \\
\text { açúcar sabor laranja em } \\
\text { envelopes (sachês). }\end{array}$ & 89 \\
\hline Senna alexandrina + Cassia fístula & $\begin{array}{l}\text { Cápsulas } 28,9 \mathrm{mg}+19,5 \\
\text { mg: embalagem com } 16 \\
\text { ou } 90 .\end{array}$ & 102 \\
\hline Senna alexandrina + Cassia fístula & $\begin{array}{c}\text { Cartucho contendo } 8 \text { ou } \\
20 \text { cápsulas. }\end{array}$ & 34 \\
\hline \multicolumn{2}{|l|}{ Total } & 257 \\
\hline
\end{tabular}

Fonte: Autores (2021).

Entre os medicamentos fitoterápicos mais vendidos para a constipação intestinal destaca-se o que tem como planta medicinal Senna alexandrina e Cassia fístula (Laboratório A) como 40\% dos medicamentos fitoterápicos para constipação entre os anos de 2019 e 2020, logo em seguida Plantago ovata (35\%), Senna alexandrina e Cassia fístula (Laboratório B) (13\%) e Aloe ferox e Gentiana lutea (12\%). Conforme pode-se observar o Gráfico 1, abaixo:

Figura 1 - Fitoterápicos para constipação intestinal mais vendidos entre agosto/2019 à agosto/2020.

\section{Fitoterápicos para constipação mais vendidos por nome de espécie vegetal}

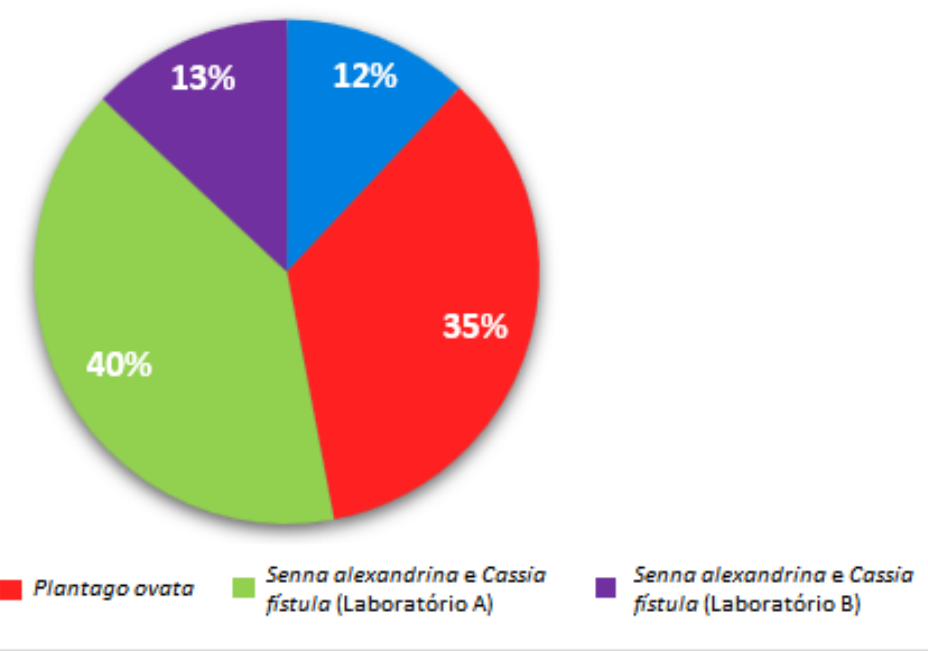

Fonte: Autores (2021). 
A demanda pode estar relacionada ao benefício que o medicamento proporciona, contudo, apesar de serem produtos fitoterápicos precisam ser usados sob orientação do farmacêutico para evitar efeitos indesejados ou riscos à saúde através do uso irracional. Um estudo apresentado por Filho et al., (2018), utilizando-se uma amostra de 117 participantes, realizou o tratamento para constipação de forma racional, com orientações e unificando o medicamento com ingestão de bastante água e prática de exercícios, evidenciando em um resultado de melhora global na constipação foi de $65,1 \%$. Esse dado embasa o uso racional desse fitoterápico e por isso aumenta a procura por esse medicamento nas drogarias.

Efeitos semelhantes foram encontrados em uma investigação Qiao et al., (2021) utilizando-se uma erva medicinal chinesa chamada de Xiaoji Dao zhi utilizada para constipação infantil. O uso apresentou-se eficiente para a evacuação dos participantes da amostra, contudo a segurança com relação ao uso de forma irracional também se apresenta obscura e se desconhecem os riscos. Portanto, a eficiência se mostrou na amostra com crianças, de maneira que estas conseguiram obter 23 evacuações por semana.

Um outro estudo semelhante realizado por Bing et al., (2020), sobre a eficiência de plantas medicinais como produtos fitoterápicos na forma farmacêutica de uso externo, constatou-se efeitos positivos para fitoterápicos que são absorvidos em uma margem de 19,97\%. Esses fitoterápicos de uso tópico para constipação são a base de Aloe emodin, rhein, crisofanol e antraquinona.

Em um estudo semelhante, realizado por Zhaohui et al., (2020) com 90 pacientes utilizando-se um medicamento fitoterápico a base da erva chinesa Byzj apresentou eficiência para a constipação, apresentando-se como uma alternativa na medicina ocidental no tratamento de pessoas com problemas relacionados à constipação. Contudo, esse mesmo medicamento apresentou efeitos adversos se utilizados a longo prazo, contribuindo com o desenvolvimento de disfunções renais.

Com relação à eficiência dos resultados apresentados pelo fitoterápico mais vendido, no caso o medicamento que tinha em sua composição Senna alexandrina e Cassia fistula, o estudo realizado por Gonçalves (2016) evidenciou que o uso da planta medicinal denominada Senna alexandrina, que faz parte da composição desse medicamento, quando extraídos os frutos e folhas, apresentam resultados eficientes na constipação intestinal que acontece de forma eventual. Esse medicamento por sua vez, apresenta 28.9 mg de Senna alexandrina Mill em sua composição.

Por outro lado, Brasil (2016) descreve algumas possíveis contraindicações do uso desse medicamento, sendo destacadas as seguintes: contraindicado para menores de 12 anos, grávidas ou lactantes, bem como para pacientes que possuem um histórico de hipersensibilidade ou alergia a qualquer componente fitoterápico. Outra contraindicação que deve ser levada em consideração é que esse medicamento não pode ser utilizado em casos crônicos de constipação e outras patologias relacionadas, tais como: distúrbios intestinais, obstrução e estenose intestinal, atonia, doenças inflamatórias intestinais. Nos casos de o indivíduo utilizar de forma contínua o que deve ser usado de forma eventual, os possíveis efeitos colaterais são: desconforto no trato intestinal, espasmos e cólicas abdominais, alteração na cor da urina para uma cor amarela escura ou marrom avermelhada. O uso irracional ou superdosagem pode causar diarreias com distúrbios hidroeletrolíticos, acidose ou alcalose metabólica, albuminúria, hematúria e hipocalemia, pode também causar a perda de peso e o desenvolvimento de caquexia. Se o paciente for idoso, pode desencadear também, hipotensão arterial ortostática, tetania e nefrite.

Na investigação de Lombardo (2021), apresentou-se uma lista de fitoterápicos que são eficientes contra a constipação, dentre eles destaca-se a Senna alexandrina, vale ressaltar que algumas plantas medicinais já foram registradas pela Anvisa e que, portanto, transmitem mais segurança quando são comercializados na indústria farmacêutica e que podem também obter em sua composição substâncias que auxiliam no tratamento de úlceras, gastrite e flatulências.

Assim, Santos (2021) trouxe para o seu estudo voltado para a avaliação do uso de Senna alexandrina observou-se que a maioria das pessoas que participaram do estudo desconheciam tanto os efeitos dessa planta quanto os benefícios, o que compreender-se que a maioria das pessoas que compram, mesmo sem prescrição médica, desconhecem os efeitos colaterais e 
todas as propriedades que o medicamento oferece, aumentando com isso o risco de superdosagem ou interferem na ação do tratamento da constipação intestinal.

Em contrapartida, realizando-se um levantamento sobre os medicamentos fitoterápicos mais vendidos, destaca-se um estudo semelhante realizado por Souza et al., (2020), na Paraíba, onde evidenciou-se que no ano de 2020, as plantas medicinais mais vendidas em medicamentos fitoterápicos foram na sua maioria em formato de cápsulas

Nessa mesma pesquisa também se evidenciou que na Paraíba, nessa farmácia de dispensação que foi pesquisada, a maioria dos fitoterápicos que foram vendidos no ano de 2020 foram mais voltados para a ação ansiolítica e para perda de peso. Além disso, a maioria dos clientes procuravam mais por cápsulas do que que outras formas farmacêuticas como xarope ou sachê. Semelhante ao resultado evidenciado nessa pesquisa, que apresentou como maior quantidade de vendas um medicamento em que sua forma de apresentação farmacêutica é em cápsulas.

Em outros estudos, Oliveira et al., (2020) apresentou os principais fitoterápicos que atuam no combate à constipação intestinal, citando como os principais tais como: Arctium lappa L. (Bardana) que se apresenta como cicatrizante e atuante em inflamações e colabora como a evacuação normal. Outra espécie é Baccharis trimera que também apresenta propriedades diuréticas. Nessa pesquisa também se evidenciou que a maioria desses fitoterápicos que atuam na constipação intestinal advém de metabólitos secundários dessas plantas.

\section{Considerações Finais}

De acordo com os resultados encontrados na pesquisa realizada no sistema de vendas da drogaria, evidenciou-se que a maior quantidade de medicamentos fitoterápicos comercializados no local pesquisado foram os utilizados para a constipação intestinal, embora exista uma grande procura por medicamentos voltados para o emagrecimento e doenças respiratórias.

Com base nisso, considera-se que mesmo sendo produtos à base de plantas medicinais, conforme discriminado nas pesquisas de casos semelhantes de estudo, evidenciou-se que existem efeitos adversos que podem surgir decorrente ao uso irracional desses medicamentos, que podem inclusive desenvolver novas desordens no organismo, principalmente quem já apresenta um histórico com outras comorbidades.

Observa-se que o medicamento mais vendido dentro da drogaria foi o medicamento que tinha em sua composição Senna alexandrina e Cassia fistula, que possui resultados positivos em outras pesquisas semelhantes, colaborando com o bom funcionamento do intestino dos pacientes, como evidenciado nas pesquisas apresentadas e nos casos estudado.

Contudo, outras pesquisas também trouxeram informações voltadas para a superdosagem, sendo nesse caso, reações adversas como diarreia e desequilíbrio da microbiota intestinal. Precisa ser levada em consideração se esse paciente também possui outras comorbidades crônicas e o uso de outros medicamentos para que as orientações possam ser mais adequadas àquele cliente, de maneira que os resultados possam ser eficientes e não comprometam a qualidade de vida deles.

Sugere-se para estudos futuros a investigação sobre todos os sintomas ou reações adversas que podem causar a automedicação, quando o cliente/paciente por iniciativa própria e sem a intervenção do farmacêutico toma altas dosagens de medicamentos fitoterápicos para constipação, evidenciando com isso os riscos ou efeitos colaterais que estes fármacos podem trazer para o uso irracional.

\section{Referências}

Basilisco, G., \& Coletta, M. (2016). Chronic constipation: a critical review. Dig Liver Dis, 45(11).

Bharucha, A. E., \& Wald, A. (2016). Funcional anorectal disorders. Gastroenterology, 130:1510-1518.

Bing, Y., Hailin, J., Jiazhen, C., Yanze, L., Xiona, L., Lijuan, H., \& Tie, L. (2021). The effectiveness of acupoint herbal patching for functional constipation. Revista Medicine, 100 (2): 349-355. 
Brasil, M. S. (2016). Política Nacional de Práticas Integrativas e Complementares no SUS - PNPIC - SUS. Ministério da Saúde, 96p.

Cieza A., et al. (2017). Towards a minimal generic set of domains of functioning and health. BMC Public, 14:218-226.

Cirillo, C., \& Capasso, R. (2015). Constipation and Botanical Medicines: An Overview. Phytother Res. 34(5): 122-134.

Colette, V. L., Araujo, C. L., \& Madruga, S. W. (2017). Prevalência e fatores associados à constipação intestinal: um estudo de base populacional em Pelotas, Rio Grande do Sul, Brasil, Cad. Saúde Pública, 26(7): 1392-402.

Dalfovo, M. S., Lana, R. A., \& Silveira, A. (2008). Métodos quantitativos e qualitativos: um resgate teórico. Revista Interdisciplinar Científica Aplicada. Blumenau, 4(2): 01-13.

Dantas, A. A. G. (2019). Constipação intestinal e funcionalidade em mulheres adultas no interior no nordeste brasileiro. https://repositorio.ufrn.br/handle/123456789/27522

Diaz, S., Bittar, K., \& Mendez, M. D. (2021). Constipação. StatPearls. Ilha do Tesouro (FL). https://www.ncbi.nlm.nih.gov/books/NBK513291/

Filho, C. I., Jung, L. K., Mallman, I. O., Sosa, F. F, Rocha, A. R., \& Bueno, P. T. B. (2018). Avaliação comparativa de eficácia clínica e tolerabilidade para a combinação de Cassia fistula e Senna alexandrina Miller em pacientes com constipação intestinal funcional crônica. Revista Soc. Bras. Clin. Med. 12(1).

Gonçalves, L. N. (2016). Levantamento etnobotânico e etnofarmacológico com raizeiros da cidade de Rio Verde - GO. Monografia de Farmácia. Universidade de Rio Verde - UniRV, Campus Rio Verde.

Huang, L., Jiang, H., \& Zhu, M. (2017). Prevalência e fatores de risco da constipação crônica entre mulheres com 50 anos ou mais em Xangai - China. Med Sci Monit, 23: 2260-7.

Jin, C., Jang, B. H., Jeon, J. P., Lee, Y. S., Yang, S. B., \& Kwon, S. (2021). Traditional East Asian herbal medicines for the treatment of poststroke constipation: A protocol for systematic review and meta-analysis. Medicine (Baltimore). 100(15):e25503.

Lombardo, M. (2021). Fitoterápicos na atenção básica de problemas gastrointestinais. Revista Ciência e Saúde, 6(1): 878-91.

Longstreth, G. F., Thompson, W.G. et al. (2016). Funcional bowel disorders. Gastroenterology, 130 (5): $1480-91$.

Pereira, A. S., et al. (2018). Metodologia da pesquisa científica. UFSM.

Qiao, L., Luo-Jia, W.,Chen ,Y., Hai-Lan,Z., \& Shu-Cheng, Z. (2021)A randomized, double-bind and placebo- controlled trial of Chinese herbal medicine in the treatment of childroon constipation. Clinical and Translational Gastroenterology, v.12, may,

Oliveira, F. M., Emerick, A. P. C., \& Soares, E. G. (2020). Aspectos epidemiológicos das doenças intestinais inflamatórias na macrorregião de saúde leste do Estado de Minas Gerais. Ciência Saúde Coletiva. 15(1):66-70.

Rad, S. (2017). Impacto dos hábitos étnicos nas medições defecográficas. Arch Iran Med, 5:115.

Santos, K. M. (2021). Avaliação dos aspectos dietéticos e investigação do uso de Senna alexandrina no tratamento de quadros de constipação intestinal. Dissertação de pós-graduação em mestrado em Ciências da Saúde. Universidade Federal de Tocantins.

Severino, A. J. (2018). Metodologia do trabalho científico. Cortez.

Severo, A. D.A. L., et al. (2018). Caracterização química e atividade biológica de Senna Alata L. Roxb e Senna Simea. Dissertação de Mestrado. Universidade Federal de Pernambuco.

Souza, L. Q. R., Maia, L. S., Sá, T. M., Trindade, Y. R. C, Silva, L. V. F, \& Castro, D. L. S. (2020). Análise dos medicamentos fitoterápicos comercializados em farmácia magistral do sertão paraíba. Research Society and Development, 9(7): 444-45.

Thompson, A. P., Wine, E., \& MacDonald, S. E. (2020). Parents' Experiences and information needs while caring for a Child with functional Constipation: A Systematic Review. Clinic Pediatric. 60(3):154-169.

Vilanova-Sanchez, A., et al. (2018). Are Senna based laxatives safe when used as long term treatment for constipation in children? J Pediatr Surg, 53(4): 722727.

Zhaohui, J., Zhengtang, L., Lei, K., Aoran, Y., Hongbo, Z., Xiaoyan, Y., Tianqing, Z., Lei, G., Aixian, L., \& Boyan, F. (2020). A randomizado double-blind placebo- controlled multicenter trial of bushen Yisui and Ziyin jiangzhuo formula for constipation in Parkinson disease. Medicine, $99(28)$ : $122-24$. 